(C) 2019 IEEE. Personal use of this material is permitted. Permission from IEEE must be obtained for all other uses, in any current or future media, including reprinting/republishing this material for advertising or promotional purposes, creating new collective works, for resale or redistribution to servers or lists, or reuse of any copyrighted component of this work in other works.

This is the accepted version of the following article: Luís Almeida, Teresa Gomes, and Carlos Henggeler Antunes. Optimization of PMU Location and Communications in a Power Grid. 2019 11th International Workshop on Resilient Networks Design and Modeling (RNDM), Nicosia, Cyprus, 1416 Oct. 2019, pp. 1-7. DOI: 10.1109/RNDM48015.2019.8949117, which has been published in final form at https://ieeexplore.ieee.org/document/8949117.

\title{
Optimization of PMU Location and Communications in a Power Grid*
}

\author{
Luís Almeida Teresa Gomes \\ Carlos Henggeler Antunes \\ University of Coimbra, Department of Electrical and Computer Engineering \\ 3030-290 Coimbra, Portugal \\ INESC Coimbra, 3030-290 Coimbra, Coimbra, Portugal \\ uc2013147802@student.uc.pt, teresa@deec.uc.pt, ch@deec.uc.pt
}

September 2019

*This work was partially supported by Fundação para a Ciência e a Tecnologia (FCT) under project grant UID/Multi/00308/2019 and is funded by ERDF Funds through the Centre's Regional Operational Program and by National Funds through the FCT - Fundação para a Ciência e a Tecnologia, I.P. under the project CENTRO-01-0145-FEDER-029312. 


\begin{abstract}
Phasor Measurement Units (PMU) are specific equipment that measure phasors, magnitude and frequency of voltage and current in key points of a power grid, allowing for a more effective management of network assets. We start by presenting some formulations for the problem of determining the location to minimize the number of PMUs installed, ensuring that all buses (nodes of the power grid) are visible by at least one PMU. The formulations take also into account the existence of Zero Injection Buses (ZIBs) and the requirement of $(\mathcal{N}-1)$ redundancy to ensure that critical buses (e.g. buses with a generator) can be observed by at least two PMUs. This guarantees, in case one out of $\mathcal{N}$ installed PMUs fails, all critical buses are still observable.

We assume that the communications network topology follows the topology of the power grid. With the goal to protect the communication infrastructure against failures and natural disasters, we propose models that minimize the number of Shared Risk Link Groups (SRLGs) and links in the path pair of each PMU to a pair of Phasor Data Concentrators (PDCs). We then modify the formulation of the PMU location problem to improve the resilience of the communication between the PMUs and the PDCs, namely by avoiding to place PMUs in buses of degree one and also by penalizing the placement of PMUs in buses that will result in path pairs to the PDCs which are not SRLG-disjoint. Results are presented to illustrate the impact of the modified PMU location model on the resilience of the communication network.

Keywords: Phasor Measurement Unit (PMU), Phasor Data Concentrator (PDC), location, Zero Injection Bus (ZIB), disaster resilience, disjoint routing, Shared Risk Link Group (SRLG).
\end{abstract}

\title{
1 Introduction
}

Installing PMUs (Phasor Measurement Units) at key points in the power grid is of paramount importance since these devices provide real time data of current and voltage phasors, magnitude and frequency in a given area, thus giving a detailed view of the grid status and power quality. One of the characteristics of good power quality is the reduction of harmful harmonics to the grid, increasing the system reliability 15. This problem is especially important in the context of the evolution of power grids to Smart Grids. According to (smartgrid.gov) a Smart Grid is a network of transmission and distribution of electricity which, in its entirety, interconnects and uses all equipment that is connected to the network (including private citizen equipment, e.g. microgenerators) to improve the quality of 
service provided, to make the grid resilient to failures and to better respond to changes of energy consumption patterns. Integrating PMUs into Smart Grids, including the communication infrastructure, is a challenge that has been a topic of research. Reference [4] analyzes the optimization of PMU location and communications infrastructure, with the goal to minimize propagation delay as well as the number of PMUs installed. In [8] and [17], in addition to the optimization of PMU location, the maximization of the communication infrastructure reliability to prevent failures in PMU observability is also considered. In [8], the authors refer to the use of the Global Positioning System (GPS) to synchronize information acquired by PMUs. An important factor affecting the communication between PMUs is the terrain topography. In [4], a proposal is presented to mitigate the negative effects of topography, considering the elevation of the terrain and the height of the towers where the communications infrastructure is installed (microwave). Similarly, [9] consider that the geography of the terrain and the coordinates of the different components that make up the WAMS (Wide Area Measurement System) are important elements to take into account.

Throughout this article we will use nodes when we speak of the communication infrastructure and buses when we speak of the PMU location. Both have the same meaning, but they are used in different contexts (Telecommunications and Energy, respectively).

\subsection{Communication between PMUs and PDCs}

There are several means of communication for PMUs. Fiber optics is used most frequently. According to [21], fiber optics is used as a means of communication between PMUs and PDCs (Phasor Data Concentrators) due to its low latency, robustness, reliability and scalability. The authors of [4] explore the advantages and disadvantages of the microwave technology to replace aerial and underground fiber optics. For this purpose, the equipment and maintenance costs are considered, as well as the terrain topography to infer possible delays in propagation. Wireless communications are gaining more and more importance, as this technology becomes more accessible. The work [7] presents an overview of the state of the art of such technologies, listing specifications such as latency, download rate and bandwidth for each wireless technology. 


\subsection{Models and optimization methods}

A formulation for the problem of optimization of PMU location and communication infrastructure as a MIQCP (Mixed Integer Quadratically Constrained Programming) model is considered in [21]. The authors consider bandwidth constraints required for the transfer of information between each CL (Communication Link), as well as the influence of Zero Injection Bus (ZIBs) and the limitations of PMUs measurement capability. By definition, a ZIB is a bus without generator and/or load and current injection, which allows buses that are adjacent to it to be visible by a PMU [21]. This visibility is justified by the Kirchhoff laws. Regarding the traffic model in a WAMS, it is comprised by PMUs, a PDC and the communication infrastructure which transmits the information through fiber optics. In [21], the authors adopted the model proposed by [19] for the estimation of the traffic generated by each PMU, considering several routes between a given PMU and PDC, as well as the bandwidth required for each connection. A PDC is an equipment that collects and synchronizes information transmitted by PMUs and possibly other PDCs in the form of phasors [2]. The cost of the communication infrastructure is estimated by summing the passive cost and the active cost. The active cost depends directly on the available bandwidth, i.e. switches and routers. The passive cost takes into account the fiber optics length, as well as the labor cost needed for the installation of the communication infrastructure. In order to generalize the cost of the CLs, the possibility of the existence of a previous CL with a given bandwidth is considered. Thus, it is possible to formulate the joint problem of PMU location and CLs, the objective function aiming to minimize the number of PMUs and the cost of the communication infrastructure that connects a PMU to a PDC, subject to the following constraints: all buses must be observed by at least least one PMU, the available bandwidth should be greater than or equal to the one of the existing CL, PMU measurement limitations (quantity of buses that a PMU can observe) and ZIBs.

An approach to minimize the use of PMUs to make a particular area observable, which may already be observed, was proposed by [3]. Bearing in mind that PMUs will be installed on power grids that are already being observed by conventional methods (Injection Measurement and Flow Measurement), the authors of [3] propose the adoption of the ABM (Augmented Bus Merging) method that relaxes the observability conditions imposed by the PMU location on a specific bus. Thus, the buses that are already observed do not need to be observed by PMUs, as the existing infrastructure serves this purpose. The objective function is the minimization of the number of PMUs installed, subject to constraints specifying that the buses are always visible according to the desired observability level (by one 
or at least two PMUs, the latter for the $(\mathcal{N}-1)$ redundancy case). The model proposed in [3] is solved using a Binary Integer Linear Problem (BILP) solver.

The problem of optimization of PMU location using a genetic algorithm, taking into account the network topology is addressed in [20]. The objective function minimizes the total number of PMUs, subject to observability and topological configuration constraints. A group of constraints based on topology (observability) is defined. A first set of constraints defines the PMUs installation on buses where they are required. The second prohibits the installation of PMUs on some buses. Once the location of a given PMU has been chosen, the third set of constraints prevents the installation of other PMUs on certain buses. Finally, for the purpose of choosing the best solutions, a redundancy constraint is introduced, which considers the total number of PMUs whose buses are observable under the Kirchhoff current law. The solutions with the highest redundancy values are the best.

In [20] it is suggested that the traditional binary genetic algorithm, used in the optimization of PMU location, has some disadvantages. That is, the search space covers all possible solutions, which can be advantageous for a low-bus configuration. However, if the number of buses grows, the number of possible solutions increases exponentially. Hence, to be possible to determine the best crossover operator configuration, it is necessary to maintain a balance between destroying admissible solutions and the difficulty of finding solutions by executing a multitude of experiences. Unfeasible solutions may be treated by methods incorporating a penalty term in the objective function that is proportional to the violation of the constraints. With the purpose of overcoming the difficulties of traditional binary genetic algorithms when applied to this type of problems, the authors propose a configuration of the algorithm in which the chromosomes correspond to the number of buses observed by a given PMU. This new configuration considers a certain probability of crossover and function-dependent mutation so that the individual with the lowest quality (fitness) is less likely to be able to reproduce and pass its characteristics to the next generation. The fitness function corresponds to the quality of the solution which may be assessed by the objective function or may include, e.g., penalty terms associated with the violation of constraints.

\subsection{Shared Risk Link Groups and disaster resilience}

An SRLG (Shared Risk Link Group) is a group of links in a communication network with the same risk of failure, i.e. connections that share one or more physical elements and, consequently, the network resources supported by these element(s) fail simultaneously if 
the event associated with the risk of failure occurs. SRLGs can also be used to represent faults geographically correlated [18]. Thus, it is up to the operator of a telecommunications network to determine the location of SRLGs that correspond to the risk of failure which they want to protect the network against. The simplest way to protect a communication from an isolated SRLG failure is to determine a pair of SRLG-disjoint paths [10] or maximally SRLG-disjoint paths when total disjointedness is not possible. A path pair is arc- and SRLG-disjoint if the working (primary) path and the protection (secondary) path share no arc nor an SRLG.

While the use of SRLG-disjoint routing increases network resilience, and in particular disaster-resilience if the SRLGs represent geographically correlated failures, it entails additional costs (paths tend to be longer and may require more expensive terminal equipment). A practical study of the increase in the path lengths and the cost of terminal equipment when considering maximally SRLG-disjoint paths and also geodiverse paths (i.e two paths separated by a given geographical distance [5]) is presented in [13]. The need for cost-efficient solutions to ensure resilient communications in the presence of disaster-based disruptions has been recognized [16]. A description of best practices for ensuring the resilience of transport networks can be found in [12] and an overview of strategies to prepare networks for large-scale natural disasters, seeking to mitigate their impact, can be found in 6 .

\subsection{Contribution}

In this work we take a lexicographical approach to the PMU location problem and resilient communications infrastructure design, considering the communication network will follow the topology of the power grid. We start by addressing the PMU location problem, not allowing PMUs to be placed in buses of degree one (unless required by double observability). This gives us the minimum number of PMUs required by the power grid to ensure total single (or double, for critical buses) visibility of each bus. Next, having previously determined the minimum number of SRLGs that will be shared by any path pair from every node to the PDCs, we can modify the formulation of the PMU location problem, by penalizing the placement of PMUs in buses that will result in path pairs to the PDCs which are not SRLG-disjoint, while keeping the maximum number of deployed PMUs limited to the minimum value previously calculated. This will improve the resilience of the communication between the PMUs and the PDCs, in particular disaster resilience if the SRLGs represent geographically correlated failures. Moreover, our results show that, re- 
laxing slightly the value of the minimum number of deployed PMUs, further improvement may be achieved.

The paper is organized as follows. In Section 2 the notation is introduced, followed in Section 3 by the formulation models for the problems to be tackled. Computational results are presented in Section 4. In Section 5 some possible future extensions of this work are outlined.

\section{Notation}

The power grid topology can be represented by a graph $G=(N, E)$, where $N$ is the set of nodes (buses) and $E$ is the set of links (undirected arcs). Each link $e$ is represented by its end nodes $\{i, j\}$, with $i, j \in N$. Each link $e=\{i, j\}$ can also be represented by a pair of symmetrical directed $\operatorname{arcs}(i, j)$ and $(j, i)$. The set of directed arcs (for communication networks) will be designated by $A$ and its elements will be indexed by $k$ (i.e $k=(i, j$ ), with $k=1, \ldots,|A|)$, where $|A|$ is the cardinal of set $A$. To simplify the notation we will also use $k^{\prime}$ to represent the symmetrical of arc $k$. The set of SRLGs in the network is represented by $\zeta$.

\subsection{Decision variables}

The formulations in section 3 will require the following variables.

- $x_{i} \in\{0,1\}$ binary variable that indicates if a PMU is installed in the bus $i$.

- $y_{i j} \in\{0,1\}$ binary variable that indicates observability due to a ZIB.

- $w_{i j} \in\{0,1\}$ binary variable that indicates if a bus $j$ is observed by a PMU installed in the bus $i$.

- $\gamma_{k} \in\{0,1\}$ binary variable that indicates if arc $k \in A$ is shared by the working and protection paths.

- $g_{r} \in\{0,1\}$ binary variable that indicates if SRLG $r \in \zeta$ is shared by the working and protection paths.

- $z_{r_{1}} \in\{0,1\}$ and $z_{r_{2}} \in\{0,1\}$ binary variables that indicate if path 1 (the active path) and path 2 (the protection path) are affected by SRLG $r$, respectively. 
- $x_{1_{k}} \in\{0,1\}$ and $x_{2_{k}} \in\{0,1\}$ binary variables that indicate whether arc $k \in A$ belongs to the active and protection path, respectively.

\subsection{Parameters}

The power grid topology and the communications network topology are described by the following parameters:

- $a_{i j}$ indicates if two buses are adjacent, forming the adjacency matrix:

$$
a_{i j}= \begin{cases}1, & \text { if bus } i \text { and bus } j \text { are connected } \\ 1, & \text { if } i=j \\ 0, & \text { otherwise }\end{cases}
$$

- $\tau_{i k}$ indicates whether an $\operatorname{arc} k \in A$ is incident on or emergent from node $i \in N$ (i.e. $\tau_{i k}$ are elements of a node-incidence matrix):

$$
\tau_{i k}=\left\{\begin{aligned}
1, & \text { if } \operatorname{arc} k \text { originates from node } i \\
-1, & \text { if arc } k \text { terminates at node } i \\
0, & \text { otherwise }
\end{aligned}\right.
$$

- $h_{r k}$ indicates if arc $k \in A$ belongs to SRLG $r \in \zeta$ :

$$
h_{r k}= \begin{cases}1, & \text { if arc } k \text { belongs to SRLG } r \\ 0, & \text { otherwise }\end{cases}
$$

- parameter $u_{i}, i \in N$ indicates if $i$ is a source, a target or an intermediate node of some flow:

$$
u_{i}=\left\{\begin{aligned}
1, & \text { if } i \text { is the source node } \\
-1, & \text { if } i \text { is the target node } \\
0, & \text { otherwise }
\end{aligned}\right.
$$

- $b_{i}, i \in N$, indicates the required level of observation of bus $i$ by the PMUs: 1 for regular buses and 2 for critical buses;

- $z_{i}, i \in N$, is the ZIB location indicator, which is 1 for buses that are ZIB and 0 otherwise. 
- $w^{\max }$ is the maximum number of buses that a PMU can observe.

- $N_{c} \subset N$ is the set of critical buses.

- $c_{k}(k \in A)$ is the cost of using arc $k$ in the communication network.

\section{Optimization models}

Before we start describing the models used, please note that a PMU installed in a given bus can observe that bus as well as those adjacent to it. Moreover, a ZIB can make buses adjacent to it observable due to the Kirchhoff current law.

In this section we will begin by describing the models used in the optimization of PMU location considering the presence of ZIBs, as well as the number of buses that a PMU can measure. When it comes to the criteria of bus observability, we will use redundancy $(\mathcal{N}-1)$ of critical buses and prohibition of PMU installation in buses, if they are not critical, with a degree of one. The degree of a bus is given by the number of buses adjacent to it (or alternatively by the number of incident links). Finally, we will use weighting coefficients to discourage the installation of PMUs in buses that have more SRLGs in common between the source and the target node. Our goal is to reorganize the location of the PMUs in the power grid, so that it will lower the number of the SRLGs in common, whilst maintaining the same number of PMUs.

\subsection{Optimization of PMU Location}

The following model includes the presence of ZIBs while guaranteeing that every bus $i$ is observed by at least $b_{i}$ PMUs as presented in [21]. The problem solved by this model is designated by $\mathcal{P}_{P M U 1}$.

$$
z_{A}=\min \sum_{i \in N} x_{i}
$$

subject to

$$
\begin{aligned}
& \sum_{j \in N} a_{i j} x_{j}+\sum_{j \in N} a_{i j} z_{j} y_{i j} \geq b_{i}, \forall i \in N \\
& \sum_{i \in N} a_{i j} y_{i j}=z_{j}, \quad \forall j \in N \\
& x_{i}, y_{i j} \in\{0,1\}, \quad \forall i, j \in N
\end{aligned}
$$


The objective function (5) is the minimization of the number of installed PMUs. Constraints (6) guarantee that each bus in the power grid is observed by $b_{i}$ PMUs, whilst also considering the extra visibility of adjacent buses of a ZIB. The constraints (7) determine the coefficients of $y_{i j}$ which compute the observability considering ZIBs.

The determination of the minimum number of PMUs, considering the number of buses which can be observed by any PMU is limited [21], is designated problem $\mathcal{P}_{P M U 2}$. The resulting model is non-linear due to constraints $(10)$, whose linearization is pre-processed by the CPLEX solver (version 12.8) [1].

$$
z_{B}=\min \sum_{i \in N} x_{i}
$$

subject to

$$
\begin{aligned}
\sum_{j \in N} a_{i j} x_{i} w_{i j} \geq b_{i}, & \forall i \in N \\
\sum_{j \in N} a_{i j} w_{i j} \leq w^{\max }, & \forall i \in N \\
\sum_{j \in N} w_{i j} \leq x_{i}, \quad & \forall i \in N \\
x_{i}, w_{i j}, y_{i j} \in\{0,1\}, \quad & \forall i, j \in N
\end{aligned}
$$

Constraints 10 indicates that every bus will be observed by at least $b_{i}$ PMUs, while limiting the total number of observable buses. Constraints (11) ensure that the number of buses that a given PMU can observe does not exceed its capacity $\left(w^{\max }\right)$. Constraints (12) guarantee that a PMU in a given bus $i$ observes a bus $j$ if there is a PMU in bus $i$ $\left(x_{i}=1\right)$.

Problem $\mathcal{P}_{P M U 3}$ determines the minimum number of PMUs to be installed taking into account the existence of ZIBs and the capacity of the PMUs. The formulation of Problem $\mathcal{P}_{P M U 3}$ combines the previous two models $\mathcal{P}_{P M U 1}$ and $\mathcal{P}_{P M U 2}$ :

$$
z_{C}=\min \sum_{i \in N} x_{i}
$$


subject to

$$
\begin{aligned}
\sum_{j \in N} a_{i j} x_{i} w_{i j}+\sum_{j \in N} a_{i j} z_{j} y_{i j} \geq b_{i}, & \forall i \in N \\
\sum_{j \in N} a_{i j} y_{i j}=z_{i}, & \forall i \in N \\
\sum_{j \in N} a_{i j} w_{i j} \leq w^{\max }, & \forall i \in N \\
\sum_{j \in N} w_{i j} \leq x_{i}, \quad & \forall i \in N \\
x_{i}, w_{i j}, y_{i j} \in\{0,1\}, & \forall i, j \in N
\end{aligned}
$$

where constraints (16), (17) and (18) are identical to constraints (7), (11) and (12), respectively.

For the adaptation of the previous model to prohibit the installation of PMUs in buses with degree one, the following constraints should be added.

$$
\begin{array}{r}
\left(\sum_{j \in N} a_{i j} x_{i} w_{i j}+\sum_{j \in N} a_{i j} z_{j} y_{i j}\right)-3 x_{i} \geq b_{i}, \\
\forall i:\left\{\begin{array}{l}
i \in N \backslash N_{c} \wedge b_{i}=2 \\
\vee \\
i \in N \wedge b_{i}=1
\end{array}\right.
\end{array}
$$

The coefficient 3 in the previous constraints results from the adjacency coefficients (1), since when $i=j, a_{i j}=1$.

Instead of introducing additional notation, from here onward, we will use designation $\mathcal{P}_{\text {PMUi }}(i=1,2,3)$ for the three problems above with the interdiction of using buses of degree one (when possible).

For the redundancy $(\mathcal{N}-1)$ in the observability of critical buses, we first need to choose the location of those buses in the power grid. To select the critical buses, we followed the criteria used in [14], namely:

- highest degree bus;

- buses with a generator;

- buses with the highest voltage. 
Once we find the location of those buses, we simply need to change the values of the observability vector $\left(b_{i}\right)$ from 1 to 2 , for the critical buses.

\subsection{Optimization of the Communication Infrastructure}

In order to be complete, a power grid observation system needs to transmit all the information acquired by the PMUs to a PDC. In this work, it is our goal to improve the resilience of the communication network between the PMUs and the PDC. With this objective in mind, we defined a pair of maximally disjoint routes between each PMU and the two PDCs. The physical medium considered for transmitting the information in the communication infrastructure is fiber optics, due to being a robust technology (not dependent on weather conditions) and able to meet the timing requirements needed by Smart Grid communications.

The routing models presented in this sub-section can contribute to improve disaster resilience: we determine pairs of maximally SRLG-disjoint paths, and the impact of high risk natural disasters in a communication network can be modelled using SRLGs.

\subsubsection{Location of PDCs}

All data measured by PMUs must be transmitted to a PDC. In the present approach we defined the location of the PDCs before determining the location of the PMUs, thus being an input to the model. It was considered that a good strategy would be to install the PDC in the centre of the network. Among several centrality measures, we chose the eigenvalue centrality method. This method consists of computing the eigenvalues of the adjacency matrix and selecting the node associated with the highest eigenvalue. According to this metric, this node is the one with greater centrality in the network, which we chose as the first PDC. The second PDC was chosen to be at two-hop minimum distance from the first PDC and having the next highest possible eigenvalue.

Consider the network transformation in Fig. 1, where we have added a fictitious node which is only connected to the PDCs. This simplifies the disjoint routes formulations as we have now a single target node (the fictitious node) to connect to every PMU via disjoint routes. The dotted lines represent possible paths between the PMU and each of the PDCs. 


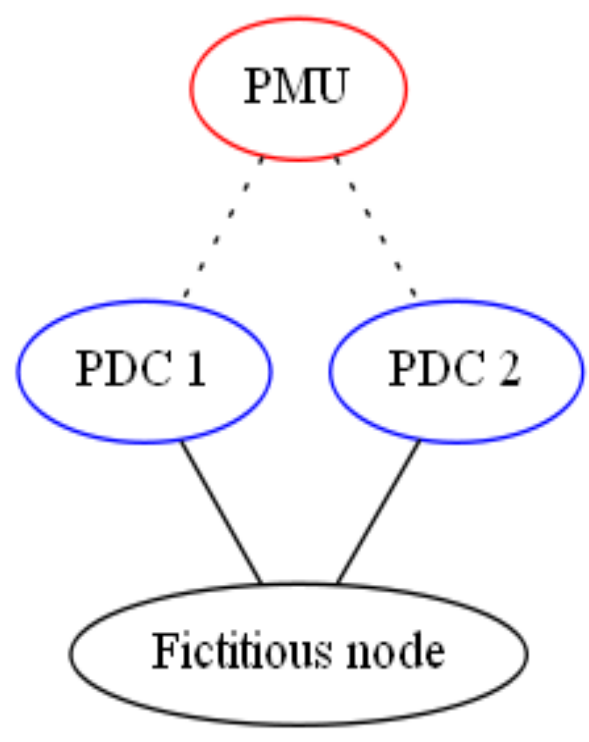

Figure 1: Connecting a PMU to the fictitious node.

\subsection{Maximally link and SRLG-disjoint paths and PMU location}

In order to make the communication infrastructure resilient, whenever possible, against single SRLG failure and against single link failures, we adapted a set of disjoint routing models described in [13]. In [13] it is assumed that the network is bi-connected. However, the topology of the power grids (IEEE N-bus) used for tests are not bi-connected due to the existence of articulation buses (nodes), bridges and spurs. Moreover, we did not consider each communication link to define its own SRLG, hence it was necessary to slightly modify the models in [13]. Namely, we needed to determine the minimum number of arcs shared by the active and protection path. This was carried out by the model given by (25)-(34), where $s$ (a bus where a PMU may be located) is the source node and $t$ is the target node (the fictitious node).

Having determined the arcs that must be used by any route from each PMU to the PDCs, this information is used to obtain the minimal number of SRLGs that must be shared by any path pair from a PMU to the PDCs (see (35)-(39)).

Our proposal is to use the topological information about the minimum number of SRLGs each node may share in the maximally disjoint path pair towards the fictitious node (i.e., the two PDCs) conditioning the location of the PMUs. For this purpose, the 
following weights are used:

$$
\begin{array}{rr}
\pi_{i}=1 /|N|, & \forall i \in N: \rho_{i t}=0 \\
\pi_{i}=\rho_{i t}, & \forall i \in N: \rho_{i t} \neq 0
\end{array}
$$

with $\rho_{i t}$ the minimum number of SRLGs shared by a path pair from node $i$ to node $t-$ objective value of (35), for $s=i$.

First, the minimum number of PMUs, $z_{C}$, is obtained by solving the formulation for problem $\mathcal{P}_{P M U 3}$, given by (14)-(20). Second, the weights $\pi_{i}$ are calculated (using the objective function value (35), determined for every node $s$ ) so we can formulate the new PMU location problem $\mathcal{P}_{P M U}$ :

$$
z_{D}=\min \sum_{i \in N} \pi_{i} x_{i}
$$

subject to constraints $(15)-(20)$ and

$$
\sum_{i \in N} x_{i} \leq z_{C}+\delta
$$

I.e., for $\delta=0$ we search for a more favorable location, from the point of view of the communication network resilience, for the minimal number of PMUs $\left(z_{c}\right)$. If $\delta>0$ it means one is willing to install more PMUs to achieve a larger number of fully disjoint route pairs.

Finally, having solved the new PMU location problem, given by (23)-(24), we can now obtain the minimal cost path pair that is maximally link and SRGL-disjoint (see (40)-(41)), from every PMU (the location of which was conditioned by the resilience requirements of the communication infrastructure) to the PDCs.

\subsection{Disjoint routing formulations}

Problem $\mathcal{P}_{C o m 1}$ seeks the minimum number of arcs in common between an active and a protection path.

$$
\eta_{s t}=\min \sum_{k \in A} \gamma_{k}
$$


subject to

$$
\begin{aligned}
\sum_{k \in A}\left(\tau_{i k} x_{1_{k}}\right)=u_{i}, & \forall i \in N \\
\sum_{k \in A}\left(\tau_{i k} x_{2_{k}}\right)=u_{i}, & \forall i \in N \\
x_{1_{k}}+x_{2_{k^{\prime}}} \leq 1, & \forall k \in A \\
x_{1_{k}}+x_{1_{k^{\prime}}} \leq 1, & \forall k \in A \\
x_{2_{k}}+x_{2_{k^{\prime}}} \leq 1, & \forall k \in A \\
\sum_{k \in A}\left(\left|\tau_{i k}\right| x_{1_{k}}\right) \leq 2, & \forall i \in N \\
\sum_{k \in A}\left(\left|\tau_{i k}\right| x_{2_{k}}\right) \leq 2, & \forall i \in N \\
x_{1_{k}}+x_{2_{k}}-\gamma_{k} \leq 1, & \forall k \in A \\
x_{1_{k}}, x_{2_{k}}, \gamma_{k} \in\{0,1\}, & \forall k \in A
\end{aligned}
$$

The objective function 25 is the minimization of the number of arcs in common between the active and protection paths, from a PMU in bus $s \in N$ to the fictitious node $t$. The constraints (26) and (27) are the usual flow conservation constraints. Constraint (28) does not allow the use of an arc and its symmetric for both the active and protection path, since we consider them to be part of the same link. Constraints (29)-(32) prevent cycles: any arc and its symmetric cannot both be in the same path; any node in either path cannot have more than two adjacent arcs in that path. Finally, constraint (33) determines if an arc $k$ must be shared by both active and protection paths (if an arc must be shared, then $\left.\gamma_{k}=1\right)$.

To make the paper self contained, next we present in detail the mentioned disjoint routing formulations, adapted from [13].

Problem $\mathcal{P}_{\text {Com } 2}$ minimizes the number of SRLGs in common in the path pair. Its formulation is:

$$
\rho_{s t}=\min \sum_{r \in \zeta} g_{r}
$$


subject to constraints $(26)-(34)$ and

$$
\begin{aligned}
l l \sum_{k \in A}\left(h_{r k} x_{1_{k}}\right) \leq|A| z_{1_{r}}, \quad & \forall r \in \zeta \\
\sum_{k \in A}\left(h_{r k} x_{2_{k}}\right) \leq|A| z_{2_{r}}, \quad & \forall r \in \zeta \\
z_{1_{r}}+z_{2_{r}}-g_{r} \leq 1, & \forall r \in \zeta \\
x_{1_{k}}, x_{2_{k}}, g_{r}, z_{1_{r}}, z_{2_{r}} \in\{0,1\}, & \forall k \in A, \forall r \in \zeta
\end{aligned}
$$

The objective function (35) is the minimization of the number of SRLGs in common in the path pair. Constraints (36) and (37) ensure that if an arc $k$ belongs to a SRLG $r$, the binary decision variables $z_{1_{r}}$ and $z_{2_{r}}$ are given the value 1 . Finally, the constraint (38) computes the number of SRLGs in common ( $g_{r}$ takes the value 1 if both $z_{1_{r}}$ and $z_{2_{r}}$ are 1). Note that constraint (38) depends on the solution obtained solving problem $\mathcal{P}_{\text {Com } 1}$, i.e. minimizing $\eta_{s t}$ (see eq. (25)).

The final problem, $\mathcal{P}_{\mathrm{Com} 3}$, is the calculation of the path pair, maximally link and SRLGdisjoint of minimum cost, from any PMU to the fictitious node $t$, which is formulated as follows:

$$
l_{s t}=\min \sum_{k \in A}\left(c_{k}\left(x_{1_{k}}+x_{2_{k}}\right)\right)
$$

subject to constraints (26)-(27), (33)-(34), (36)- (39) and

$$
\rho_{s t}=\sum_{r \in \zeta} g_{r}
$$

The objective function (40) is the minimization of the path cost of a link-disjoint path pair with minimum number of shared arcs and SRLGs. Since we can take different paths, with the same number of SRLGs in common, between the origin and target nodes we should not fix the $\operatorname{arcs} k$ in which there are SRLGs in common (if $\rho_{s t}>0$ ). Therefore, we need to use the constraint (41) to guarantee the total minimum number of SRLGs in common given by the resolution of problem $\mathcal{P}_{\text {Com } 2}$ while we minimize the path cost.

\section{Computational results}

The models presented in the previous section were applied to the IEEE 14-bus, 30-bus, 57-bus, 118-bus, 300-bus power grids [22] - these are tranmission grids. 
Table 1: Some topology information, where $\alpha_{i}$ is the degree of bus $i \in N$ and $\bar{\alpha}$ is the average bus degree

\begin{tabular}{lccccc}
\hline IEEE System & $|N|$ & $|E|$ & $\min _{i \in N} \alpha_{i}$ & $\max _{i \in N} \alpha_{i}$ & $\bar{\alpha}$ \\
\hline IEEE 14-bus & 14 & 20 & 1 & 5 & 2.9 \\
IEEE 30-bus & 30 & 41 & 1 & 7 & 2.7 \\
IEEE 57-bus & 57 & 78 & 1 & 6 & 2.7 \\
IEEE 118-bus & 118 & 179 & 1 & 9 & 3.0 \\
IEEE 300-bus & 300 & 409 & 1 & 11 & 2.7 \\
\hline
\end{tabular}

Table 2: Critical buses

\begin{tabular}{lccc}
\hline IEEE System & Generators & $\begin{array}{c}\text { Highest } \\
\text { Degree } \\
\text { Bus }\end{array}$ & $\begin{array}{c}\text { Highest } \\
\text { Voltage } \\
\text { Buses }\end{array}$ \\
\hline IEEE 14-bus & $1-2-3-6-8$ & 4 & 0 \\
IEEE 30-bus & $1-2-13-23$ & 6 & 0 \\
IEEE 57-bus & $1-2-3-6-8-9-12$ & 9 & 0 \\
IEEE 118-bus & Omitted (54) & 49 & Omitted (11) \\
IEEE 300-bus & Omitted (69) & 268 & Omitted (14) \\
\hline
\end{tabular}

Some information about the topology of these systems can be seen in Table 1. These power grids are often used in the literature to test different approaches to the problem of optimization of PMU location and communication infrastructure. Using the open-source software MATPOWER developed by [22, it was possible to process the data necessary for the creation of the grids, namely the adjacency and incidence matrix, the location of the ZIBs and critical buses (Table 2). Once the data needed to test the models were obtained, we used the CPLEX solver to compute the solution for the different models.

Whilst processing the data for the power grids using MATPOWER, namely for the location of critical buses, some of those buses that were generators were also ZIBs (although, by definition, a ZIB is a bus without generator and/or load). Hence, we only included in Table 2 generators that were not in nodes identified as ZIBs. Furthermore, regarding the capacity of PMUs to observe buses, we used $w^{\max }=100$ [21].

The resolution of the problems $\mathcal{P}_{P M U 3}$ - see eqs. (14)-(20) - resulted in the minimum number of PMUs shown in Table 3 .

For each IEEE power grid, 10 groups of SRLGs were randomly generated. These SRLGs had to pass certain conditions, namely: the maximum and minimum number of links per SRLG was 8 and 2, respectively, and the maximum number of SRLGs per link was 4. 
Table 3: Minimal number of PMUs, considering ZIBs

IEEE System Single Obs. Double Obs. for $i \in N_{c}$

\begin{tabular}{lcc}
\hline IEEE14 & 3 & 6 \\
IEEE30 & 7 & 9 \\
IEEE57 & 11 & 13 \\
IEEE118 & 28 & 47 \\
IEEE300 & 68 & 112 \\
\hline
\end{tabular}

The SRLG generation starts by randomly selecting a link, and then randomly selecting a link among those adjacent to the links in the SRLG under construction until it reaches the desired dimension (previously randomly generated) - uniformly distributed random numbers were used. The links in each SRLG were selected so the sub-graph associated with each SRLG was connected, as no GPS location for the nodes was provided. SRLGs correspond to multiple failure scenarios, which can be used to model and evaluate the impact of regional failures [18].

Figures 2 and 3 present, for those networks, the results for single and double observability of the PMUs. The label "k-S" corresponds to $\sum_{i \in N: x_{i}=1} \rho_{i t}$, which is the total number of SRLGs shared by the installed PMUs, and "N-S" is the number of installed PMUs for which it was not possible to obtain a fully SRLG-disjoint active and protection path to the PDCs. The columns labels, " $i$-Obs", " $i$-Obs-W", " $i$-Obs-W- $\delta$ ", have the following meaning:

- $i=1,2$ for single and double observation of every critical bus, repectively;

- $W$ : the objective function was $z_{D}$ (placement of the PMUs, influenced by the shared SRLGs);

- $\delta=1,2$ in (24), is the additional number of PMUs in excess of the minimum value given by $z_{C}$ and used in " $i$-Obs-W".

As expected, the number of PMUs with SRLGs in common increases from single to double observation of critical PMUs, due to the larger number of PMUs installed.

Regarding the effectiveness of the proposed strategy for PMU placement considering the requirements of the communication network infrastructure, it results in a lower number of shared SRLGs (more significant for the larger networks) and also in a lower number of PMUs that do not have a fully SRLG-disjoint path pair towards the PDCs. Although allowing the minimal value of PDCs to increase by one unit, it still allows to find alternative 
locations for the PMUs thus contributing to a more resilient communication network, the improvement stalls for $\delta=2$ (results are identical to $\delta=1$ in the case of Fig. 2, but a slight improvement can still be observed in Fig. 3 for some IEEE power grids).

In fact for the IEEE 118-bus power grid, the use of the weights defined in eq. (21) and (22) resulted in a decrease from an average of 19.2 SRLGs shared by PMUs to 16.3 and 12.8 , for $\delta=0$ and $\delta=1$, respectively, which is a significant relative decrease. Moreover, the average number of PMUs with fully disjoint routes to the PDCs increased from to 14.8 to 16.7 and 20.6 for $\delta=0$ and $\delta=1$, respectively. For both parameters, the relative variation was significant. In the case of $(\mathcal{N}-1)$ redundancy for critical bus (i.e. the bus must have double observation), the improvements are less impressive, but still significant: the average of 38 SRLGs shared by PMUs was reduced to 32.9 and 30.4 , for $\delta=0$ and $\delta=1$, respectively; also the average number of PMUs with fully disjoint routes to the PDCs increased from 22.5 to 25.7 and 27.8 (14\% and $23 \%$ increase, respectively), for $\delta=0$ and $\delta=1$, respectively.

\section{Conclusion}

In this article we present a new approach for solving the problem of optimization of PMU location and communication infrastructure. Unlike most relevant articles on this topic, in addition to the optimization of the PMU location, we have also optimized the communication infrastructure to make it more resilient against failures and natural disasters, which can be modeled using SRLGs.

It was proposed to condition the location of the PMUs to buses that allow to minimize the total number of SRLGs in common (for maximally disjoint routes from the PMU to the PDC). After computing the minimum number of PMUs that ensure that all buses are visible, and in the case of $(\mathcal{N}-1)$ redundancy that all critical buses can be observed by at least two PMUs, the PMU location problem is solved once again. The placement of a PMU in a bus is then penalized, if from that bus it is not possible to reach the PMU through a fully SRLG-disjoint path pair, while limiting the maximum number of PMUs to install. Results using five IEEE N-bus power grids, namely in the case of the IEEE 118-bus network, showed that for the larger networks significant improvements were obtained, regarding both the number of shared SRLGs and the number of PMUs with fully SRLG-disjoint routes.

Regarding future work, it was noticed that the topology of the power grids used does 


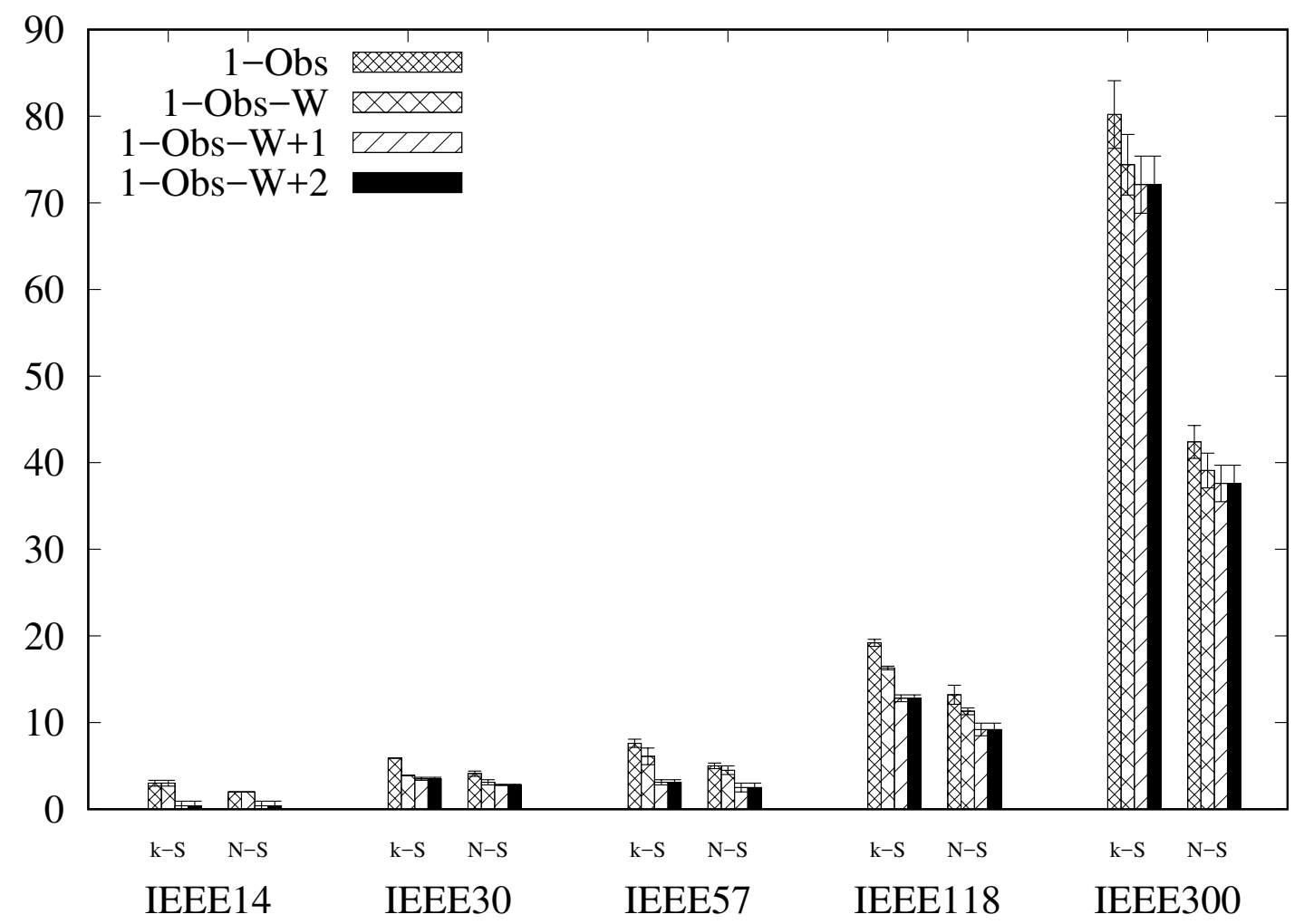

Figure 2: Number of shared SRLGs (k-S) and number of nodes for which no SRLG fullydisjoint path pair was found to the two PDCs (N-S); both 1-Obs and 1-Obs-W find the location of the minimum number of PMUs ensuring all buses are visible by at least one PMU; 1-Obs-W seeks to place PMUs in buses with minimal number of SRLGs in common; " +1 " and " +2 " indicate the number of allowed PMUs could exceed the minimum by 1 and 2 units, respectively. 


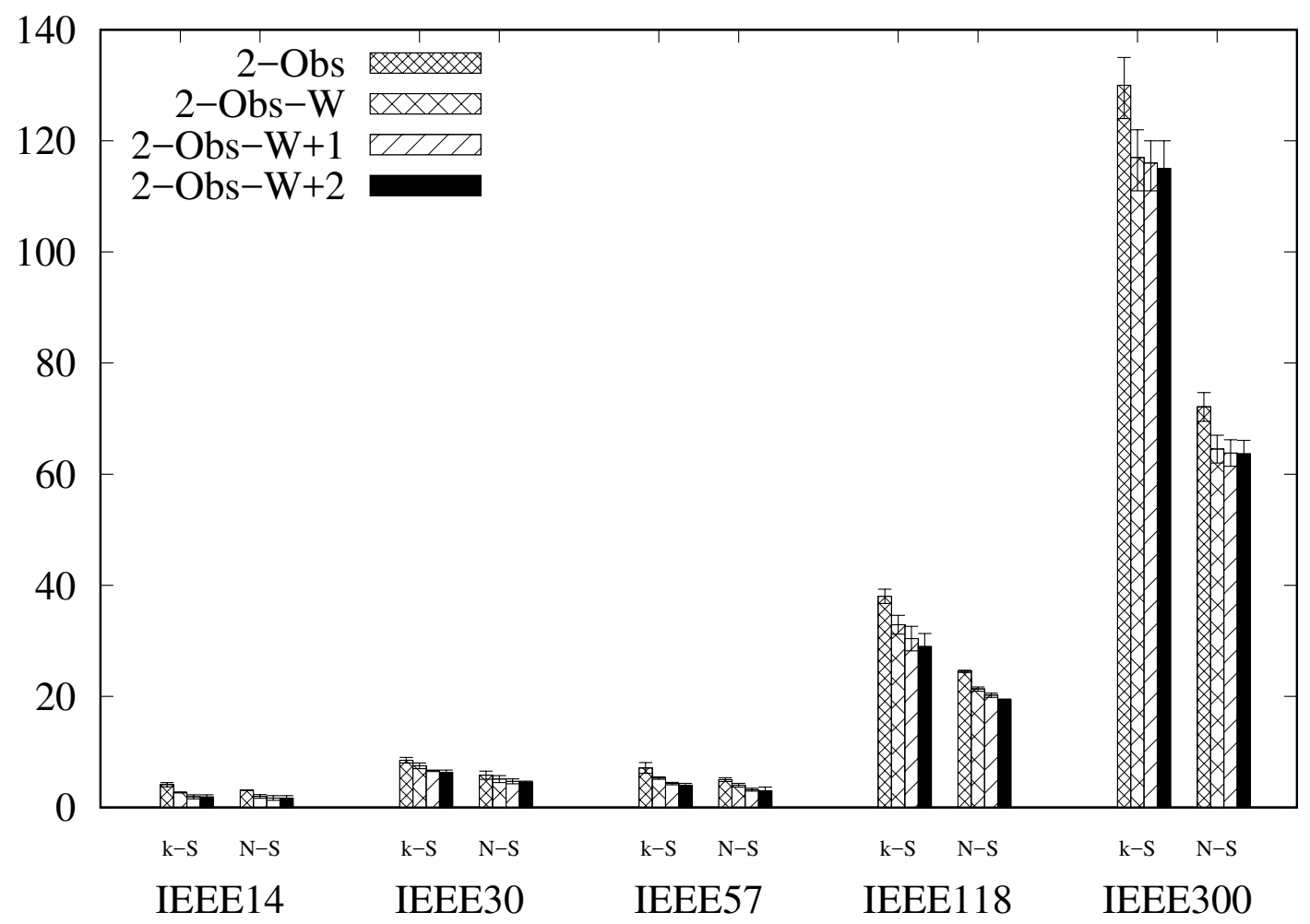

Figure 3: Number of shared SRLGs (k-S) and number of nodes for which no SRLG fullydisjoint path pair was found to the two PDCs (N-S); both 2-Obs and 2-Obs-W find the minimum number of locate PMUs ensuring all buses are visible by at least one PMU and that critical buses are observed by at leat two PMUs; 2-Obs-W seeks to place PMUs in buses with minimal number of SRLGs in common; "+1" and "+2" indicate the number of allowed PMUs could exceed the minimum by 1 and 2 units, respectively. 
not allow to obtain link-disjoint paths for several PMUs (due to the existence of buses of degree one, bridges and spurs). Hence, the communication network topology needs to be augmented with respect to the topology of the power grid (as in [11]). In the present work, disjoint end-to-end routes were considered. However, having determined the location of the PMUs, the routes to the PDC could be solved as a Steiner tree problem to reduce the use of communication resources. To improve resilience a pair of maximally link and SRLG disjoint trees should be calculated. Additionally one could consider jointly the PMU and PDC location problems.

\section{Acknowledgment}

The authors would like to thank Álvaro Gomes (University of Coimbra \& INESC Coimbra) for sharing his expertise regarding power networks. This article is based on research developed in the framework of COST Action CA15127 (Resilient communication services protecting end-user applications from disaster-based failures, RECODIS), supported by COST (European Cooperation in Science and Technology) and project ESGRIDS - Enhancing Smart Grids for Sustainability (SAICTPAC/0004/2015- POCI-01-0145-FEDER-016434).

\section{References}

[1] IBM ILOG CPLEX Optimization Studio V12.8. IBM, 20117.

[2] IEEE Guide for Phasor Data Concentrator Requirements for Power System Protection, Control, and Monitoring. IEEE Std C37.244-2013, pages 1-65, May 2013.

[3] N. H. Abbasy and H. M. Ismail. A unified approach for the optimal PMU location for power system state estimation. IEEE Transactions on Power Systems, 24(2):806-813, May 2009.

[4] B. Appasani and D. K. Mohanta. Co-Optimal Placement of PMUs and Their Communication Infrastructure for Minimization of Propagation Delay in the WAMS. IEEE Transactions on Industrial Informatics, 14(5):2120-2132, May 2018.

[5] A. de Sousa, D. Santos, and P. Monteiro. Determination of the minimum cost pair of D-geodiverse paths. In The 2017 International Conference on Design of Reliable Communication Networks (DRCN 2017), Munich, March 8-10 2017. 
[6] T. Gomes, J. Tapolcai, C. Esposito, D. Hutchison, F. Kuipers, J. Rak, A. de Sousa, A. Iossifides, R. Travanca, J. André, L. Jorge, L. Martins, P. O. Ugalde, A. Pašić, D. Pezaros, S. Jouet, S. Secci, and M. Tornatore. A survey of strategies for communication networks to protect against large-scale natural disasters. In 8th International Workshop on Resilient Networks Design and Modeling (RNDM), pages 11-22, Sept 2016.

[7] R. N. Gore and S. P. Valsan. Wireless communication technologies for smart grid (wams) deployment. In 2018 IEEE International Conference on Industrial Technology (ICIT), pages 1326-1331, Feb 2018.

[8] X. Huang, J. Liu, Y. Liu, L. Xu, J. Yang, and Y. Ni. A GA based optimized PMUlocation-decision algorithm considering WAMS reliability. In 2014 International Conference on Power System Technology, pages 433-439, Oct 2014.

[9] E. Inga, R. Hincapié, C. Paida, and S. Espinosa. Optimal geographic placement of PMU for Wide Area Measurement System. In 2016 IEEE Ecuador Technical Chapters Meeting (ETCM), pages 1-6, Oct 2016.

[10] Jian Qiang Hu. Diverse routing in optical mesh networks. IEEE Transactions on Communications, 51(3):489-494, March 2003.

[11] V. Kounev, M. Lévesque, D. Tipper, and T. Gomes. Reliable communication networks for smart grid transmission systems. Journal of Network and Systems Management, 24(3):629-652, 2016.

[12] A. Mauthe, D. Hutchison, E. K. Çetinkaya, I. Ganchev, J. Rak, J. P. G. Sterbenz, M. Gunkel, P. Smith, and T. Gomes. Disaster-resilient communication networks: Principles and best practices. In 2016 8th International Workshop on Resilient Networks Design and Modeling (RNDM), pages 1-10, Sept 2016.

[13] B. Nedic, M. Gunkel, T. Gomes, and R. Girão-Silva. SRLG-disjointness and geodiverse routing - a practical network study and operational conclusions. In 10th International Workshop on Resilient Networks Design and Modeling (RNDM), pages 1-8, Longyearbyen - Svalbard (Spitsbergen), Norway, August 27-29 2018.

[14] A. Pal, G. A. Sanchez-Ayala, V. A. Centeno, and J. S. Thorp. A PMU Placement Scheme Ensuring Real-Time Monitoring of Critical Buses of the Network. IEEE Transactions on Power Delivery, 29(2):510-517, April 2014. 
[15] L. T. C. Pulz, C. Baumgarten, R. C. Leborgne, and F. H. García. Harmonic distortion assessment using state estimation algorithm. In 2016 17th International Conference on Harmonics and Quality of Power (ICHQP), pages 757-761, Oct 2016.

[16] J. Rak, D. Hutchison, E. Calle, T. Gomes, M. Gunkel, P. Smith, J. Tapolcai, S. Verbrugge, and L. Wosinska. Recodis: Resilient communication services protecting enduser applications from disaster-based failures. In 18th International Conference on Transparent Optical Networks (ICTON), July 10-14 2016. Invited paper.

[17] D. R. Shrivastava, S. A. Siddiqui, and K. Verma. Optimal PMU placement for coordinated observability of power system under contingencies. In 2017 IEEE International Conference on Circuits and Systems (ICCS), pages 334-339, Dec 2017.

[18] J. Tapolcai, L. Rónyai, B. Vass, and L. Gyimóthi. List of shared risk link groups representing regional failures with limited size. In IEEE INFOCOM 2017 - IEEE Conference on Computer Communications, pages 1-9, May 2017.

[19] M. H. F. Wen and V. O. K. Li. Optimal phasor data concentrator installation for traffic reduction in smart grid wide-area monitoring systems. In 2013 IEEE Global Communications Conference (GLOBECOM), pages 2622-2627, Dec 2013.

[20] Yuanyuan Zhao, Peng Yuan, Qian Ai, and Tianguang Lv. Optimal PMU placement considering topology constraints. International Journal of Electrical Power \& Energy Systems, 73:240 - 248, 2015.

[21] X. Zhu, M. H. F. Wen, V. O. K. Li, and K. Leung. Optimal PMU-Communication Link Placement for Smart Grid Wide-Area Measurement Systems. IEEE Transactions on Smart Grid, 10(4):4446-4456, July 2019.

[22] R. D. Zimmerman, C. E. Murillo-Sanchez, and R. J. Thomas. Matpower: Steady-state operations, planning, and analysis tools for power systems research and education. IEEE Transactions on Power Systems, 26(1):12-19, Feb 2011. 